\title{
TyG Index Distributions and Relationship to Cardiometabolic Risk Factors in Children and Adolescents: A Population-Based Study
}

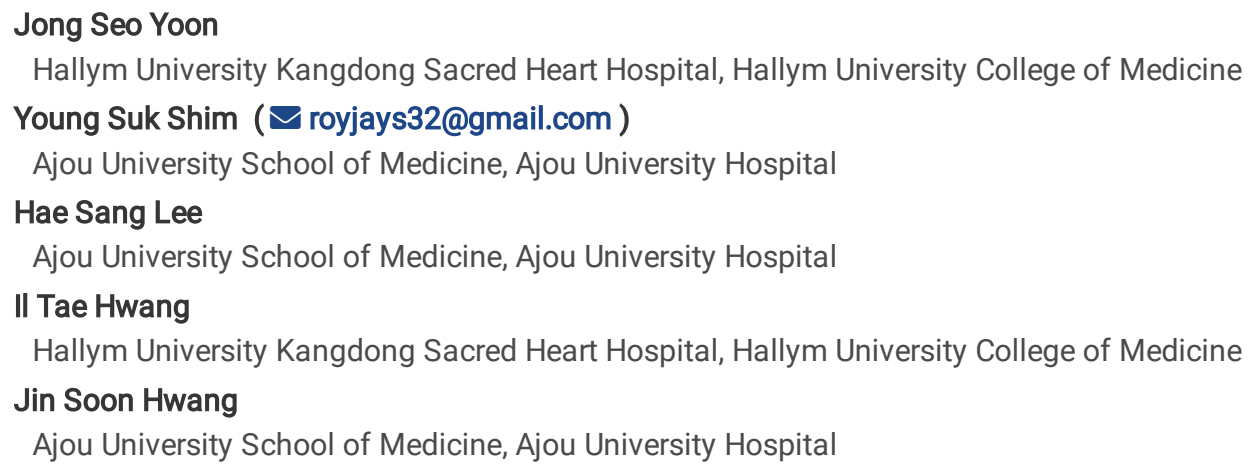

\section{Research Article}

Keywords: index, glucose, linear, triglyceride, relationship

Posted Date: July 21st, 2021

DOl: https://doi.org/10.21203/rs.3.rs-721138/v1

License: (c) (i) This work is licensed under a Creative Commons Attribution 4.0 International License. Read Full License 


\section{Abstract}

The purpose of this study was to present age- and sex-specific distributions of the triglyceride-glucose (TyG) index and to evaluate their relationship with cardiometabolic risk factors in children and adolescents. A total of 7,404 participants aged 10-18 years from the Korean National Health and Nutrition Survey were included as the reference population. The TyG index was calculated as $\ln ($ fasting triglyceride $[\mathrm{mg} / \mathrm{dL}]$ $x$ fasting glucose $[\mathrm{mg} / \mathrm{dL}] / 2$ ). The percentile of the TyG index exhibited a steady linear relationship with age for both sexes. TyG index significantly correlated with waist circumference (WC) standard deviation score (SDS; $r=0.110, p<0.001$ ), systolic blood pressure (SBP; $r=$ $0.104, p<0.001)$, diastolic blood pressure (DBP; $r=0.083, p<0.001)$, glucose $(r=0.220, p<0.001)$, high-density lipoprotein cholesterol (HDL-C; $r=-0.325, p<0.001$ ), and triglycerides (TG; $r=0.926, p<0.001$ ). Multivariate linear regression analysis revealed that the TyG index was significantly associated with WC SDS $(\beta=0.116, p<0.001), \operatorname{SBP}(\beta=2.009, p<0.001), \operatorname{DBP}(\beta=1.464, p<0.001)$, glucose $(\beta=3.376, p<0.001)$, HDL-C $(\beta=-6.431, p<0.001)$, and TG $(\beta=85.518, p<0.001)$. Our results suggest that the TyG index has a steady linear distribution for sex and age in children and adolescents and constitutes an indicator for predicting metabolic disorders that could lead to cardiovascular disease later in life.

\section{Introduction}

Cardiovascular disease (CVD), a leading cause of morbidity and mortality, is a global disease burden ${ }^{1}$. Hyperglycemia, central obesity, elevated triglycerides, decreased high-density lipoprotein (HDL), and elevated blood pressure (BP) are components of metabolic syndrome (MetS), and each factor may independently increase the risk of CVD 2,3 . These risk factors can even cluster in children and increase the risk of CVD, eventually leading to the onset of CVD in youth ${ }^{4,5}$. Therefore, the early identification of high-risk groups and lifestyle modifications are necessary to prevent disease progression. Insulin resistance (IR) plays a major role in the pathogenesis of CVD ${ }^{6}$. The homeostatic model assessment for insulin resistance (HOMA-IR), a commonly used method for evaluating IR, is a clinical predictor of the development of MetS, type 2 diabetes mellitus (T2DM), and CVD in youth and adults ${ }^{7-11}$. However, it is difficult to assess IR in children and adolescents because of physiological characteristics that temporarily increase IR during puberty ${ }^{12,13}$. HOMA-IR in pediatric populations is not evenly distributed by sex, age, and BMI standard deviation score (SDS), and there is no consensus on the normal reference range. Therefore, there is currently no consensus for diagnosing diseases related to IR and evaluating disease status.

The TyG index has emerged as a reliable surrogate marker for IR. The TyG index is significantly associated with the presence of T2DM, nonalcoholic fatty liver disease (NAFLD), and MetS in adults and is a better predictor of IR than HOMA-IR ${ }^{14-18}$. Recent studies have reported that the TyG index can predict the presence and progression of coronary artery calcification (CAC) in adults and is associated with cardiovascular risk factors in children and adolescents ${ }^{19-22}$. In many studies in children and adolescents, cutoff points for the TyG index for MetS and cardiometabolic risk have been suggested, and its usefulness has been sufficiently emphasized. For the TyG index to be useful in clinical practice, it is necessary to determine its association with various confounding factors and whether it is consistently distributed in children and adolescents; however, studies on these characteristics are still lacking. A detailed distribution of the TyG index in children and adolescents has not yet been presented, and the effects of clinical confounders, such as age, sex, and BMI SDS, are unknown.

Therefore, this study aimed to present a detailed distribution and curve for the TyG index in children and adolescents and investigate whether confounding factors affect its ability to evaluate cardiometabolic risk factors.

\section{Results}

\section{Clinical characteristics of the study population}

The clinical characteristics of the reference population (3,945 boys and 3,459 girls) are presented as mean values \pm standard deviation or frequency lrb\%) (Table 1). The mean ages of the boys and girls were $13.77 \pm 2.50$ and $13.85 \pm 2.51$ years, respectively. Height SDS, weight SDS, SBP, DBP, and serum biochemistry results were higher in boys than in girls $(P<0.01)$. The mean TyG index was higher in boys $(8.09 \pm 0.52)$ than in girls $(8.15 \pm 0.48)(P<0.001)$. Boys were more likely to be alcohol drinkers and smokers and engage in a higher frequency of physical activity than girls (all $P<0.01$ ). 
Table 1

Clinical characteristics of study population $(n=7,404)$

\begin{tabular}{|c|c|c|c|}
\hline & Boys $(n=3,945)$ & Girls $(n=3,459)$ & $P$ \\
\hline Age, y & $13.77 \pm 2.50$ & $13.85 \pm 2.51$ & 0.169 \\
\hline Height SDS & $0.80 \pm 1.06$ & $0.53 \pm 1.04$ & $<0.001$ \\
\hline Weight SDS & $0.43 \pm 1.04$ & $0.30 \pm 1.05$ & $<0.001$ \\
\hline BMI SDS & $0.11 \pm 1.08$ & $0.10 \pm 1.06$ & 0.681 \\
\hline WC SDS & $-0.17 \pm 1.13$ & $-0.14 \pm 1.0$ & 0.189 \\
\hline $\mathrm{SBP}, \mathrm{mmHg}$ & $108.69 \pm 10.65$ & $104.10 \pm 9.28$ & $<0.001$ \\
\hline DBP, mmHg & $66.49 \pm 9.55$ & $65.50 \pm 8.30$ & $<0.001$ \\
\hline Glucose, mg/dL & $90.54 \pm 7.16$ & $89.24 \pm 8.15$ & $<0.001$ \\
\hline $\mathrm{T}-\mathrm{C}, \mathrm{mg} / \mathrm{dL}$ & $155.30 \pm 26.97$ & $163.17 \pm 26.15$ & $<0.001$ \\
\hline $\mathrm{HDL}-\mathrm{C}, \mathrm{mg} / \mathrm{dL}$ & $49.80 \pm 9.89$ & $52.11 \pm 9.86$ & $<0.001$ \\
\hline $\mathrm{TG}, \mathrm{mg} / \mathrm{dL}$ & $82.93 \pm 47.34$ & $86.49 \pm 44.09$ & 0.001 \\
\hline LDL-C, mg/dL & $88.92 \pm 23.21$ & $93.76 \pm 22.80$ & $<0.001$ \\
\hline TyG index & $8.09 \pm 0.52$ & $8.15 \pm 0.48$ & $<0.001$ \\
\hline Alcohol drinker (\%) & $219(5.55)$ & $135(3.90)$ & 0.001 \\
\hline Smoker (\%) & $621(15.74)$ & $234(6.76)$ & $<0.001$ \\
\hline Household income $\leq 1$ st quartile (\%) & $435(11.03)$ & $384(11.10)$ & 0.948 \\
\hline Rural residence (\%) & 655 (16.60) & $577(16.68)$ & 0.953 \\
\hline Physically active (\%) & $2094(53.08)$ & $1510(43.65)$ & $<0.001$ \\
\hline
\end{tabular}

Reference interval of the TyG index in the study population using the LMS method

Percentiles and L, M, and S values of the TyG index by sex and age for the study population aged 10-18 years are presented in Table 2 . The TyG index distribution charts for all participants, boys, and girls were stably distributed (Fig. 1a, 1b, and 1c, respectively). The median TyG index values of the boys and girls remained nearly constant at approximately 8.07 and 8.14, respectively. In girls, the TyG index exhibited a steady linear distribution across all ages and a steady linear decrease from 11 to 18 years. In boys, the TyG index also presented a steady linear distribution at all ages and a steady increase at an age below the 50th percentile. For all participants, the median TyG index remained nearly constant at approximately 8.11. The TyG index exhibited a steady linear distribution across all ages and a slight decline from 11 to 18 years over the 50th percentile. 
Table 2

Reference interval of the triglyceride-glucose (TyG) index in the study population according to the LMS method

\begin{tabular}{|c|c|c|c|c|c|c|c|c|c|c|c|c|c|c|c|}
\hline \multicolumn{16}{|c|}{ All participants } \\
\hline \multirow[t]{2}{*}{ Age } & \multirow[t]{2}{*}{$n$} & \multirow[t]{2}{*}{$\mathrm{L}$} & \multirow[t]{2}{*}{$M$} & \multirow[t]{2}{*}{$S$} & \multicolumn{11}{|c|}{ Percentile } \\
\hline & & & & & 3rd & 5 th & 10th & 15th & 25th & 50th & 75th & 85th & 90th & 95th & 97th \\
\hline 10 & 814 & -0.210 & 8.104 & 0.064 & 7.184 & 7.293 & 7.465 & 7.583 & 7.761 & 8.104 & 8.467 & 8.670 & 8.811 & 9.027 & 9.172 \\
\hline 11 & 895 & -0.210 & 8.119 & 0.063 & 7.210 & 7.319 & 7.488 & 7.605 & 7.780 & 8.119 & 8.477 & 8.677 & 8.816 & 9.029 & 9.171 \\
\hline 12 & 882 & -0.210 & 8.125 & 0.062 & 7.228 & 7.335 & 7.502 & 7.617 & 7.791 & 8.125 & 8.477 & 8.674 & 8.811 & 9.020 & 9.159 \\
\hline 13 & 913 & -0.210 & 8.119 & 0.061 & 7.236 & 7.341 & 7.506 & 7.619 & 7.790 & 8.119 & 8.465 & 8.658 & 8.793 & 8.998 & 9.135 \\
\hline 14 & 929 & -0.210 & 8.105 & 0.060 & 7.237 & 7.341 & 7.503 & 7.614 & 7.782 & 8.105 & 8.445 & 8.635 & 8.768 & 8.969 & 9.103 \\
\hline 15 & 800 & -0.210 & 8.092 & 0.060 & 7.237 & 7.339 & 7.499 & 7.609 & 7.774 & 8.092 & 8.426 & 8.612 & 8.742 & 8.940 & 9.071 \\
\hline 16 & 752 & -0.210 & 8.081 & 0.059 & 7.240 & 7.340 & 7.498 & 7.606 & 7.768 & 8.081 & 8.409 & 8.592 & 8.720 & 8.913 & 9.043 \\
\hline 17 & 762 & -0.210 & 8.073 & 0.058 & 7.245 & 7.344 & 7.499 & 7.606 & 7.765 & 8.073 & 8.396 & 8.576 & 8.701 & 8.891 & 9.018 \\
\hline 18 & 657 & -0.210 & 8.069 & 0.057 & 7.253 & 7.351 & 7.504 & 7.608 & 7.766 & 8.069 & 8.386 & 8.563 & 8.686 & 8.873 & 8.998 \\
\hline \multicolumn{16}{|l|}{ Boys } \\
\hline \multirow[t]{2}{*}{ Age } & $n$ & $\mathrm{~L}$ & $M$ & $S$ & \multicolumn{11}{|c|}{ Percentile } \\
\hline & & & & & $3 r d$ & 5th & 10th & 15th & 25th & 50th & 75th & 85th & 90th & 95th & 97th \\
\hline 10 & 439 & -0.306 & 8.025 & 0.067 & 7.085 & 7.196 & 7.371 & 7.491 & 7.673 & 8.025 & 8.400 & 8.611 & 8.758 & 8.984 & 9.136 \\
\hline 11 & 482 & -0.306 & 8.038 & 0.066 & 7.110 & 7.220 & 7.392 & 7.511 & 7.690 & 8.038 & 8.407 & 8.614 & 8.759 & 8.982 & 9.131 \\
\hline 12 & 483 & -0.306 & 8.051 & 0.065 & 7.136 & 7.244 & 7.414 & 7.531 & 7.708 & 8.051 & 8.414 & 8.618 & 8.761 & 8.979 & 9.126 \\
\hline 13 & 493 & -0.306 & 8.064 & 0.064 & 7.161 & 7.268 & 7.436 & 7.552 & 7.726 & 8.064 & 8.421 & 8.622 & 8.762 & 8.977 & 9.121 \\
\hline 14 & 492 & -0.306 & 8.076 & 0.063 & 7.186 & 7.292 & 7.458 & 7.572 & 7.744 & 8.076 & 8.428 & 8.626 & 8.764 & 8.975 & 9.116 \\
\hline 15 & 438 & -0.306 & 8.089 & 0.062 & 7.211 & 7.315 & 7.479 & 7.592 & 7.761 & 8.089 & 8.436 & 8.630 & 8.766 & 8.973 & 9.112 \\
\hline 16 & 380 & -0.306 & 8.102 & 0.061 & 7.236 & 7.339 & 7.500 & 7.611 & 7.779 & 8.102 & 8.443 & 8.635 & 8.768 & 8.972 & 9.108 \\
\hline 17 & 383 & -0.306 & 8.115 & 0.060 & 7.261 & 7.362 & 7.522 & 7.631 & 7.796 & 8.115 & 8.450 & 8.639 & 8.770 & 8.971 & 9.105 \\
\hline 18 & 355 & -0.306 & 8.127 & 0.059 & 7.285 & 7.386 & 7.543 & 7.651 & 7.813 & 8.127 & 8.458 & 8.644 & 8.773 & 8.970 & 9.102 \\
\hline \multicolumn{16}{|l|}{ Girls } \\
\hline \multirow[t]{2}{*}{ Age } & $n$ & $\mathrm{~L}$ & $M$ & $S$ & \multicolumn{11}{|c|}{ Percentile } \\
\hline & & & & & $3 r d$ & 5th & 10th & 15th & 25th & 50th & 75th & 85th & 90th & 95th & 97th \\
\hline 10 & 375 & -0.381 & 8.191 & 0.060 & 7.339 & 7.439 & 7.597 & 7.706 & 7.871 & 8.191 & 8.530 & 8.720 & 8.852 & 9.053 & 9.186 \\
\hline 11 & 413 & -0.381 & 8.216 & 0.059 & 7.369 & 7.469 & 7.626 & 7.734 & 7.898 & 8.216 & 8.553 & 8.741 & 8.872 & 9.071 & 9.204 \\
\hline 12 & 399 & -0.381 & 8.212 & 0.058 & 7.373 & 7.472 & 7.627 & 7.734 & 7.896 & 8.212 & 8.545 & 8.731 & 8.860 & 9.057 & 9.188 \\
\hline 13 & 375 & -0.381 & 8.179 & 0.058 & 7.351 & 7.449 & 7.602 & 7.708 & 7.868 & 8.179 & 8.507 & 8.690 & 8.818 & 9.012 & 9.141 \\
\hline 14 & 437 & -0.381 & 8.135 & 0.057 & 7.319 & 7.415 & 7.566 & 7.671 & 7.829 & 8.135 & 8.458 & 8.638 & 8.764 & 8.954 & 9.081 \\
\hline 15 & 362 & -0.381 & 8.088 & 0.057 & 7.285 & 7.380 & 7.529 & 7.631 & 7.787 & 8.088 & 8.406 & 8.584 & 8.707 & 8.894 & 9.019 \\
\hline 16 & 372 & -0.381 & 8.047 & 0.056 & 7.256 & 7.349 & 7.496 & 7.597 & 7.750 & 8.047 & 8.360 & 8.535 & 8.656 & 8.841 & 8.963 \\
\hline 17 & 379 & -0.381 & 8.021 & 0.056 & 7.239 & 7.331 & 7.477 & 7.577 & 7.728 & 8.021 & 8.330 & 8.502 & 8.622 & 8.804 & 8.924 \\
\hline 18 & 302 & -0.381 & 8.013 & 0.055 & 7.240 & 7.331 & 7.474 & 7.573 & 7.723 & 8.013 & 8.319 & 8.489 & 8.607 & 8.787 & 8.906 \\
\hline
\end{tabular}


Correlations between the TyG index and clinical variables, including sex; age; BMI SDS; WC SDS; SBP; DBP; glucose, T-C, HDL-C, TG, LDL-C levels; alcohol drinking; smoking; household income; rural residence; and physical activity, are presented in Table 3. In the adjusted Pearson's correlation analyses, after controlling for sex, age, and BMI SDS in all participants (adjusted model 2), the TyG index was positively correlated with WC SDS ( $r=0.110, P<0.001)$; SBP $(r=0.104, P<0.001)$; DBP $(r=0.083, P<0.001)$; and glucose $(r=0.220, P<0.001)$, T-C $(r=0.241, P<$ 0.001), TG ( $r=0.926, P<0.001)$, and LDL-C $(r=0.051, P<0.001)$ levels, but negatively correlated with HDL-C levels $(r=-0.325, P<0.001)$. After controlling for age and BMI SDS, the correlations between the TyG index and the above variables in boys and girls were similar to the above results (adjusted model 4). 
Table 3

Unadjusted and adjusted correlations between the triglyceride-glucose (TyG) index and cardiometabolic risk factors in the study population ( $n$ $=7,404$ )

\begin{tabular}{|c|c|c|c|c|c|c|}
\hline & \multicolumn{2}{|c|}{ Unadjusted model } & \multicolumn{2}{|c|}{ Adjusted model 1} & \multicolumn{2}{|c|}{ Adjusted model 2} \\
\hline & $\mathbf{r}$ & $P$ & $r$ & $P$ & $r$ & $P$ \\
\hline Sex (girls) & 0.051 & $<0.001$ & - & - & - & - \\
\hline Age, y & -0.035 & 0.002 & - & - & - & - \\
\hline BMI SDS & 0.246 & 0.001 & 0.245 & $<0.001$ & - & - \\
\hline WC SDS & 0.267 & $<0.001$ & 0.266 & $<0.001$ & 0.110 & $<0.001$ \\
\hline SBP, mmHg & 0.141 & $<0.001$ & 0.169 & $<0.001$ & 0.104 & $<0.001$ \\
\hline DBP, mmHg & 0.086 & $<0.001$ & 0.106 & $<0.001$ & 0.083 & $<0.001$ \\
\hline Glucose, mg/dL & 0.240 & $<0.001$ & 0.244 & $<0.001$ & 0.220 & $<0.001$ \\
\hline $\mathrm{T}-\mathrm{C}, \mathrm{mg} / \mathrm{dL}$ & 0.273 & $<0.001$ & 0.266 & $<0.001$ & 0.241 & $<0.001$ \\
\hline $\mathrm{HDL}-\mathrm{C}, \mathrm{mg} / \mathrm{dL}$ & -0.350 & $<0.001$ & -0.365 & $<0.001$ & -0.325 & $<0.001$ \\
\hline $\mathrm{TG}, \mathrm{mg} / \mathrm{dL}$ & 0.931 & $<0.001$ & 0.931 & $<0.001$ & 0.926 & $<0.001$ \\
\hline \multirow[t]{3}{*}{ LDL-C, mg/dL } & 0.098 & $<0.001$ & 0.091 & $<0.001$ & 0.051 & $<0.001$ \\
\hline & \multicolumn{2}{|c|}{ Unadjusted model } & \multicolumn{2}{|c|}{ Adjusted model 3} & \multicolumn{2}{|c|}{ Adjusted model 4} \\
\hline & $\mathrm{R}$ & $P$ & $r$ & $P$ & $r$ & $P$ \\
\hline Age, y & 0.060 & $<0.001$ & - & - & - & - \\
\hline BMI SDS & 0.276 & $<0.001$ & 0.282 & $<0.001$ & - & - \\
\hline WC SDS & 0.301 & $<0.001$ & 0.303 & $<0.001$ & 0.117 & $<0.001$ \\
\hline SBP, mmHg & 0.187 & $<0.001$ & 0.178 & $<0.001$ & 0.089 & $<0.001$ \\
\hline DBP, mmHg & 0.133 & $<0.001$ & 0.119 & $<0.001$ & 0.089 & $<0.001$ \\
\hline Glucose, mg/dL & 0.210 & $<0.001$ & 0.224 & $<0.001$ & 0.192 & $<0.001$ \\
\hline $\mathrm{T}-\mathrm{C}, \mathrm{mg} / \mathrm{dL}$ & 0.292 & $<0.001$ & 0.308 & $<0.001$ & 0.268 & $<0.001$ \\
\hline HDL-C, mg/dL & -0.340 & $<0.001$ & -0.336 & $<0.001$ & -0.286 & $<0.001$ \\
\hline $\mathrm{TG}, \mathrm{mg} / \mathrm{dL}$ & 0.928 & $<0.001$ & 0.928 & $<0.001$ & 0.922 & $<0.001$ \\
\hline \multirow[t]{3}{*}{ LDL-C, mg/dL } & 0.105 & $<0.001$ & 0.115 & $<0.001$ & 0.055 & $<0.001$ \\
\hline & \multicolumn{2}{|c|}{ Unadjusted model } & \multicolumn{2}{|c|}{ Adjusted model 3} & \multicolumn{2}{|c|}{ Adjusted model 4} \\
\hline & $r$ & $P$ & $r$ & $P$ & $r$ & $P$ \\
\hline Age, y & -0.154 & $<0.001$ & - & - & - & - \\
\hline BMI SDS & 0.209 & $<0.001$ & 0.207 & $<0.001$ & - & - \\
\hline WC SDS & 0.222 & $<0.001$ & 0.228 & $<0.001$ & 0.100 & $<0.001$ \\
\hline SBP, mmHg & 0.113 & $<0.001$ & 0.128 & $<0.001$ & 0.082 & $<0.001$ \\
\hline DBP, mmHg & 0.027 & 0.117 & 0.067 & $<0.001$ & 0.049 & 0.004 \\
\hline
\end{tabular}

BMI, body mass index; SDS, standard deviation score; WC, waist circumference; SBP, systolic blood pressure; DBP, diastolic blood pressure; T-C, total cholesterol; HDL-C, high-density lipoprotein cholesterol; TG, triglycerides; LDL, low-density lipoprotein cholesterol.

Adjusted model 1: adjusted for sex and age.

Adjusted model 2: adjusted for sex, age, and BMI SDS.

Adjusted model 3: adjusted for age.

Adjusted model 4: adjusted for age and BMI SDS. 


\begin{tabular}{|c|c|c|c|c|c|c|}
\hline & \multicolumn{2}{|c|}{ Unadjusted model } & \multicolumn{2}{|c|}{ Adjusted model 1} & \multicolumn{2}{|c|}{ Adjusted model 2} \\
\hline & $\mathbf{r}$ & $P$ & $\mathbf{r}$ & $P$ & $\mathbf{r}$ & $P$ \\
\hline Glucose, mg/dL & 0.287 & $<0.001$ & 0.267 & $<0.001$ & 0.251 & $<0.001$ \\
\hline $\mathrm{T}-\mathrm{C}, \mathrm{mg} / \mathrm{dL}$ & 0.239 & $<0.001$ & 0.238 & $<0.001$ & 0.227 & $<0.001$ \\
\hline $\mathrm{HDL}-\mathrm{C}, \mathrm{mg} / \mathrm{dL}$ & -0.383 & $<0.001$ & -0.382 & $<0.001$ & -0.350 & $<0.001$ \\
\hline $\mathrm{TG}, \mathrm{mg} / \mathrm{dL}$ & 0.934 & $<0.001$ & 0.933 & $<0.001$ & 0.930 & $<0.001$ \\
\hline LDL-C, mg/dL & 0.078 & $<0.001$ & 0.079 & $<0.001$ & 0.057 & $<0.001$ \\
\hline
\end{tabular}

BMI, body mass index; SDS, standard deviation score; WC, waist circumference; SBP, systolic blood pressure; DBP, diastolic blood pressure; T-C, total cholesterol; HDL-C, high-density lipoprotein cholesterol; TG, triglycerides; LDL, low-density lipoprotein cholesterol.

Adjusted model 1: adjusted for sex and age.

Adjusted model 2: adjusted for sex, age, and BMI SDS.

Adjusted model 3: adjusted for age.

Adjusted model 4: adjusted for age and BMI SDS.

\section{Association between the TyG index and cardiometabolic risk factors on multiple linear regression analysis}

Results of the multiple linear regression analysis of the TyG index and WC SDS; SBP; DBP; and glucose, T-C, HDL-C, TG, and LDL-C levels are shown in Table 4. After controlling for sex, age, BMI SDS, alcohol drinking, smoking, household income, rural residence, and physical activity among all participants, WC $(\beta=0.116, P<0.001)$; SBP $(\beta=2.009, P<0.001)$; DBP $(\beta=1.464, P<0.001)$; and glucose $(\beta=3.376, P<0.001)$, T-C $(\beta$ $=13.012, P<0.001)$, HDL-C $(\beta=-6.431, P<0.001)$, TG $(\beta=85.518, P<0.001)$, and LDL-C $(\beta=2.339, P<0.001)$ levels were significantly associated with the TyG index. In boys and girls, multiple linear regression analysis after controlling for age, BMI SDS, alcohol drinking, smoking, household income, rural residence, and physical activity showed that these cardiometabolic risk factors were also significantly associated with the TyG index, similar to the results for the whole population. 
Table 4

Association between the triglyceride-glucose (TyG) index and cardiometabolic risk factors using multiple linear regression analysis in the study population $(n=7,404)$

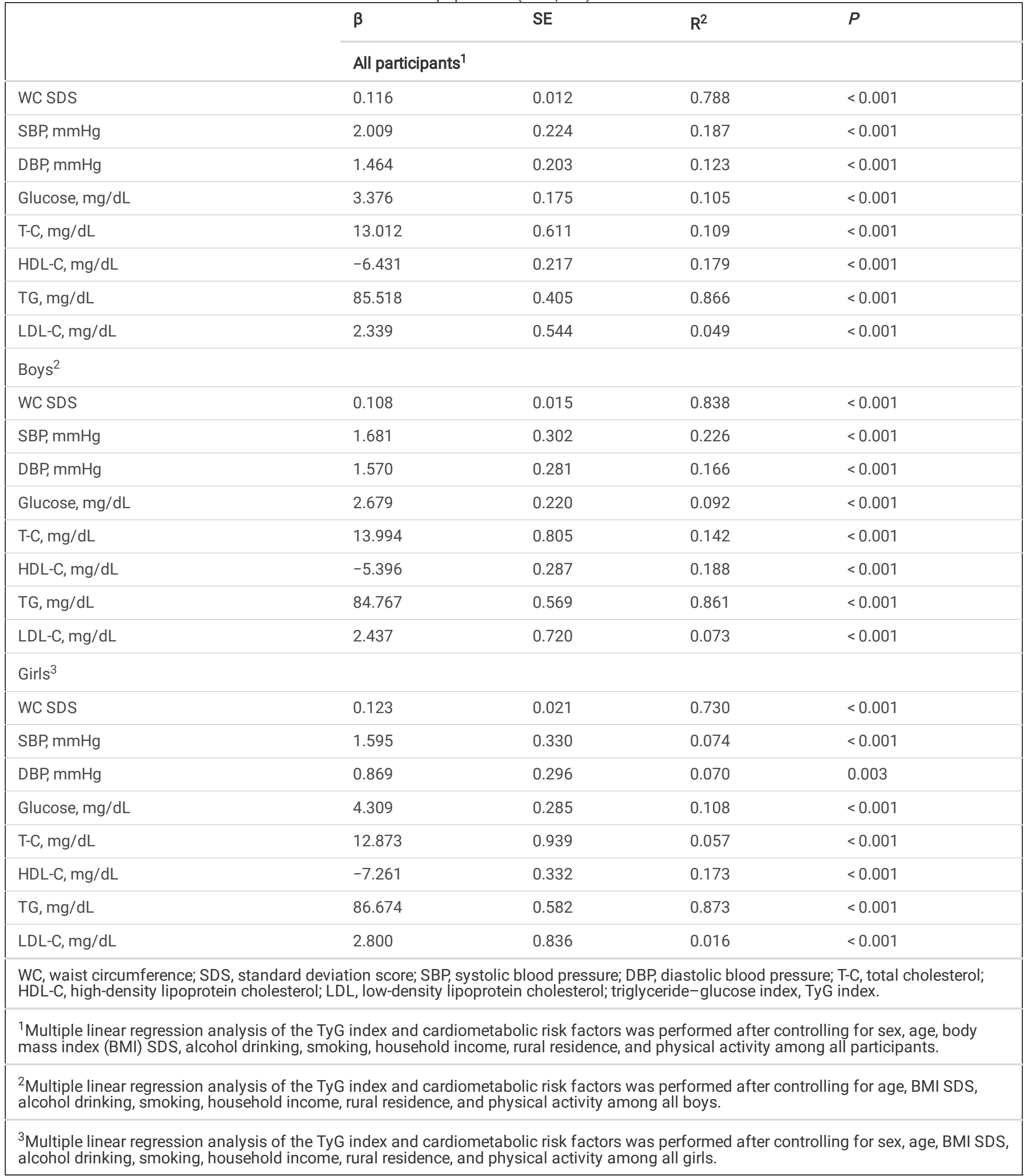

\section{Discussion}


The main finding of the present study was that the TyG index showed a steady linear distribution in both boys and girls and differed significantly by sex. The TyG index was independently significantly associated with WC SDS; SBP; DBP; and glucose, T-C, TG, LDL-C, and HDL-C levels after adjustment for sex, age, and BMI SDS.

With the increase in childhood obesity worldwide, so does the incidence of MetS and T2DM ${ }^{23-25}$. These problems persist until adulthood, leading to complications such as T2DM and CVD caused by MetS; therefore, it is necessary to prevent disease progression using early detection. Obesity induces IR, which is the common component of the shared pathology of MetS, T2DM, and CVD; therefore, it is necessary to measure IR early in overweight and obese children 26,27 . The hyperinsulinemic euglycemic clamp is the gold standard method of insulin resistance measurement; however, it is time-consuming and expensive, limiting its clinical use ${ }^{28}$. Fasting plasma insulin, HOMA-IR, and QUICKI are the most commonly used insulin-resistant surrogate markers ${ }^{7}$. These tests are currently useful for evaluating IR; however, they have the following limitations in youth: 1) a reliable reference range has not yet been proposed because it is difficult to determine normal values and cutoff points due to a lack of longitudinal evidence in youth for predicting cardiovascular outcome risk and 2) the values vary according to pubertal development and ethnic differences ${ }^{8}$. In addition, universal insulin assay standardization is lacking because of variable insulin measurement techniques among laboratories ${ }^{29}$.

The TyG index has recently emerged as a surrogate marker for IR ${ }^{15,30-33}$. In adults, it is useful for predicting T2DM, MetS, NAFLD, and CVD and is superior to the HOMA-IR index. Many studies have evaluated the relationship between the TyG index and CVD. The TyG index is independently associated with the presence and progression of CAC, which is an effective marker for atherosclerosis-related CVD ${ }^{34}$. Although the mechanism underlying the association between the TyG index level and CVD risk factors remains unclear, IR is a major contributor to CVD, and the TyG index is a reliable surrogate marker for IR, which may indirectly explain this relationship. An elevated TyG index is significantly related to elevated BP in healthy children aged 6-15 years ${ }^{35}$. In Brazilian children aged 4-9 years, overweight, total body fat and central fat, increased BP, and worsening lipid profile were associated with an increased TyG index. Although atherosclerotic CV events rarely occur in youth, atherogenic processes that lead to CVD begin in childhood and progress throughout life ${ }^{5}$. Therefore, establishing healthy behaviors for the heart and managing CV risk factors in childhood may prevent or delay the development of atherosclerosis and, in turn, reduce the risk of CVD later in life.

One of the reasons why it is difficult to assess cardiometabolic risk factors in children and adolescents is that the criteria are varied and differ slightly, as suggested by Cook, de Ferranti, and the International Diabetes Federation ${ }^{36}$. In the present study, cardiometabolic risk factors, such as central obesity, hypertension, dyslipidemia, and hyperglycemia, known independent risk factors for CVD, were independently associated with the TyG index after controlling for confounding factors such as age, sex, and BMI. Therefore, using only fasting blood glucose and TG levels, the TyG index enables a comprehensive and simple assessment of several cardiovascular risk factors in children and adolescents.

Although the TyG index is certainly a reliable and useful test for detecting IR-related diseases such as CVD, it is necessary to confirm its limitations in childhood and adolescence, such as with the HOMA-IR, before applying it to clinical practice. In this study, detailed distributions and smooth curves using the LMS method were presented for boys, girls, and all participants aged 10-18 years. The TyG index showed a stable distribution that differed from the previously reported distribution for HOMA-IR. The conventional IR evaluation method using insulin could not provide a diagnostic value for pediatric IR and a value that can represent disease progression because IR fluctuates greatly with confounding factors such as age, sex, and BMI. The difference in the distribution of surrogate markers using insulin is that the TyG index in the present study showed a stable distribution, even though there was a distribution difference between boys and girls. In future studies, it will be necessary to explore the effect of the difference in distribution between boys and girls in determining the cutoff value for predicting IR-related diseases. If the difference in distribution between the sexes does not affect the TyG index cutoff value, the previously reported TyG cutoff values will provide reliable values for clinical application and will be useful for assessing disease progression in children and adolescents in the future.

The results of the present study are valuable as a large nationally representative study population, but there are some limitations. First, because the data obtained from the KNHANES does not contain information about Tanner stage or sex hormones levels and does not provide data for children under 10 years of age, the differences in TyG index between pubertal and prepubertal periods cannot be compared. The comparison of TyG index distributions between the prepubertal and pubertal periods is thus required in further research. Despite these limitations, our results are meaningful as a practical reference for tracking children and adolescents aged $>10$ years, the age at which pediatricians evaluate IR-related diseases. Second, because we analyzed the cross-sectional data of only Korean children and adolescents, our results cannot be directly applicable to other races. Therefore, we propose further research in other less homogeneous countries to determine the characteristics of the curves and distributions of the TyG index in different ethnic groups.

Although the TyG index is recognized as a good surrogate marker for indirectly measuring IR and is thought to be useful and convenient in children and adolescents, its characteristics have not been studied with respect to various clinical variables. To the best of our knowledge, this study is the first to analyze the TyG index characteristics related to clinical variables and to evaluate the characteristics of the TyG index

Page 9/13 
distribution in children and adolescents. Here, we evaluated the value of the TyG index as a surrogate insulin resistant marker that can overcome the limitations of IR assessment caused by differences in the physical characteristics with age in children and adolescents.

In conclusion, this pediatric population-based study confirmed that the TyG index has clinical value by showing a stable distribution in children and adolescents regardless of age, sex, and BMI. In addition, this study proved that the TyG index is a surrogate marker for insulin resistance because it can individually or comprehensively evaluate various cardiovascular risk factors based only on fasting blood sugar and TG levels without the complexity of evaluating risk factors by considering sex, age, and BMI SDS.

\section{Methods}

\section{Study population}

Statistical analyses were performed using data obtained from the Korea National Health and Nutrition Examination Survey (KNHANES) 20082016; a total of 81,503 subjects were registered. The KNHANES, a population-based cross-sectional survey, was designed to assess the health and nutritional status of a nationally representative non-institutionalized Korean sample ${ }^{37}$. This survey used a stratified multilevel probability sampling method to select participants by household units. The Korean national survey consists of three parts: a health questionnaire, a health checkup, and a nutritional assessment. We included 9,287 children and adolescents aged 10-18 years in the preliminary analyses. Participants for whom anthropometric variables or health questionnaire responses $(n=685)$ or BMI and BP data $(n=1,186)$ were unavailable were excluded. We also excluded participants with triglyceride $(T G)$ levels $\geq 400 \mathrm{mg} / \mathrm{dL}(n=13)$ because the low-density lipoprotein cholesterol (LDL-C) level was determined using Friedewald's equation. Finally, 7,417 participants aged 10-18 years were included in this study. All data sets from the KNHANES are publicly accessible at http://knhanes.cdc.go.kr. The study protocol for the KNHANES 2008-2016 was approved by the Institutional Review Board of the Korean Centers for Disease Control and Prevention. Informed consent was obtained from all KNHANES participants and their legal guardians. The KNHANES was performed in accordance with relevant guidelines and regulations. The present study was approved by the Institutional Review Board of Hallym University Kangdong Sacred Heart Hospital (IRB no. KANGDONG 2021-01-006).

\section{Measurements}

Height, weight, WC, SBP, and DBP were determined according to standardized protocols. BMI was calculated as the ratio of weight to height squared $\left(\mathrm{kg} / \mathrm{m}^{2}\right)$. The SDS values for height, weight, WC, and BMI were assessed using the LMS (L, lambda for the Box-Cox power for skewness; $\mathrm{M}$, mu for the median; $\mathrm{S}$, sigma for the generalized coefficient of variation) method (SDS = [measured value/M] ${ }^{1 / L} / \mathrm{LS}$ ) based on the 2017 Korean national reference ${ }^{38}$. SBP and DBP $(\mathrm{mmHg})$ were evaluated in the right upper arm three times using a calibrated sphygmomanometer (Baumanometer Desk model 0320, Baum, NY, USA) and an appropriately sized cuff. Measurements were conducted at 2min intervals. The mean of the last two values was used as SBP and DBP in the analyses.

Blood samples were obtained from the antecubital vein after the participants had fasted for at least $8 \mathrm{~h}$. The collected blood samples were analyzed within $24 \mathrm{~h}$ in a central laboratory (NeoDin Medical Institute, Seoul, Korea). Biochemical tests, including glucose, total cholesterol (TC), HDL cholesterol (HDL-C), and TG levels, were performed using an automatic analyzer (Hitachi 7600, Hitachi, Tokyo, Japan). The LDL-C level $(\mathrm{mg} / \mathrm{dL})$ was calculated using Friedewald's Eq. $3^{9}$. The TyG index was calculated as follows: In(fasting TG [mg/dL] $\times$ fasting glucose $[\mathrm{mg} / \mathrm{dL}] / 2)^{30}$

\section{Collection of socioeconomic characteristics and medical history}

Lifestyle-related variables included alcohol consumption, smoking, physical activity, household income, and residence. Alcohol drinkers were defined as boys and girls who consumed two or more alcoholic beverages per month in the previous year, and participants were divided into two groups (drinkers versus non-drinkers). Smokers were defined as those who smoked five or more packs of cigarettes during their lifetime, and participants were divided into two groups (smokers versus nonsmokers). Physical activity was defined as one or more of the following: (i) vigorous physical activity for at least 3 days for 20 minutes per week; (ii) moderate physical activity, at least 5 days for 30 minutes per week; and (iii) light physical activity, walking for at least 5 days for 30 minutes per week. Subjects were divided into two groups according to their participation in physical activity (yes or no). Household income is presented in quartiles, and the subjects were divided into two groups (lowest quartile or second lowest quartile and above). Participants' areas of residence were divided into two groups (urban versus rural).

\section{Statistical analysis}

R version 3.6.1 for Windows (The R Foundation for Statistical Computing, Vienna, Austria) was used to perform the statistical analyses. The parameters of LMS for the TyG index were determined using the GAMLSS package ${ }^{40}$. The reference interval for the sex-and age-specific TyG index was obtained using the LMS method and is presented as the following percentile values: 3rd, 5th, 10th, 15th, 25th, 50th, 75th, 85th, 90th, 95th, and 97th percentiles. To determine statistical significance, analysis of variance and chi-squared tests were used to examine continuous and categorical variables according to sex-and age-specific TyG index groups. Continuous variables and categorical variables were presented 
as mean (standard deviation) and percentages (\%). Unadjusted and adjusted Pearson's correlation analyses were performed to investigate the correlation between the TyG index and clinical variables. In all participants, adjusted models included controls for sex and age (model 1) and for sex, age, and BMI SDS (model 2). In boys and girls, adjusted models 3 and 4 included controls for age, age, and BMI SDS, respectively. In all participants, to evaluate the association between the TyG index and cardiometabolic risk factors, such as WC SDS; SBP; DBP; and glucose, T-C, HDL-C, TG, and LDL-C levels, multiple linear regression analyses were conducted after control for sex, age, BMI SDS, alcohol drinking, smoking, physical activity, and rural residence.

\section{References}

1. Roth, G. A. et al. Global, Regional, and National Burden of Cardiovascular Diseases for 10 Causes, 1990 to 2015. J Am Coll Cardiol, 70, 125 https://doi.org/10.1016/j.jacc.2017.04.052 (2017).

2. Ahrens, W. et al. Metabolic syndrome in young children: definitions and results of the IDEFICS study. Int J Obes (Lond) 38 Suppl 2, S4-14, doi:10.1038/ijo.2014.130 (2014).

3. Yusuf, S. et al. Effect of potentially modifiable risk factors associated with myocardial infarction in 52 countries (the INTERHEART study): case-control study., 364, 937-952 https://doi.org/10.1016/S0140-6736(04)17018-9 (2004).

4. Chu, N. F., Rimm, E. B., Wang, D. J., Liou, H. S. \& Shieh, S. M. Clustering of cardiovascular disease risk factors among obese schoolchildren: the Taipei Children Heart Study. Am J Clin Nutr, 67, 1141-1146 https://doi.org/10.1093/ajcn/67.6.1141 (1998).

5. de Ferranti, S. D. et al. Cardiovascular Risk Reduction in High-Risk Pediatric Patients: A Scientific Statement From the American Heart Association., 139, e603-e634 https://doi.org/10.1161/CIR.0000000000000618 (2019).

6. Ginsberg, H. N. Insulin resistance and cardiovascular disease. J Clin Invest, 106, 453-458 https://doi.org/10.1172/JCl10762 (2000).

7. Tagi, V. M., Giannini, C. \& Chiarelli, F. Insulin Resistance in Children. Front Endocrinol (Lausanne), 10, 342 https://doi.org/10.3389/fendo.2019.00342 (2019).

8. Yin, J. et al. Insulin resistance determined by Homeostasis Model Assessment (HOMA) and associations with metabolic syndrome among Chinese children and teenagers. Diabetol Metab Syndr, 5, 71 https://doi.org/10.1186/1758-5996-5-71 (2013).

9. Gast, K. B., Tjeerdema, N., Stijnen, T., Smit, J. W. \& Dekkers, O. M. Insulin resistance and risk of incident cardiovascular events in adults without diabetes: meta-analysis. PLoS One, 7, e52036 https://doi.org/10.1371/journal.pone.0052036 (2012).

10. Isokuortti, E. et al. Use of HOMA-IR to diagnose non-alcoholic fatty liver disease: a population-based and inter-laboratory study., 60, 18731882 https://doi.org/10.1007/s00125-017-4340-1 (2017).

11. Katsuki, A. et al. Homeostasis model assessment is a reliable indicator of insulin resistance during follow-up of patients with type 2 diabetes., 24, 362-365 https://doi.org/10.2337/diacare.24.2.362 (2001).

12. Pilia, S. et al. The effect of puberty on insulin resistance in obese children. J Endocrinol Invest, 32, 401-405 https://doi.org/10.1007/BF03346475 (2009).

13. Peplies, J. et al. Percentiles of fasting serum insulin, glucose, HbA1c and HOMA-IR in pre-pubertal normal weight European children from the IDEFICS cohort. Int J Obes (Lond), 38 (Suppl 2), S39-47 https://doi.org/10.1038/ijo.2014.134 (2014).

14. Khan, S. H. et al. Metabolic clustering of risk factors: evaluation of Triglyceride-glucose index (TyG index) for evaluation of insulin resistance. Diabetol Metab Syndr, 10, 74 https://doi.org/10.1186/s13098-018-0376-8 (2018).

15. Mohd Nor, N. S., Lee, S., Bacha, F., Tfayli, H. \& Arslanian, S. Triglyceride glucose index as a surrogate measure of insulin sensitivity in obese adolescents with normoglycemia, prediabetes, and type 2 diabetes mellitus: comparison with the hyperinsulinemic-euglycemic clamp. Pediatr Diabetes, 17, 458-465 https://doi.org/10.1111/pedi.12303 (2016).

16. Low, S. et al. The role of triglyceride glucose index in development of Type 2 diabetes mellitus. Diabetes Res Clin Pract, 143, $43-49$ https://doi.org/10.1016/j.diabres.2018.06.006 (2018).

17. Hameed, E. K. TyG index a promising biomarker for glycemic control in type 2 Diabetes Mellitus. Diabetes Metab Syndr, 13, 560-563 https://doi.org/10.1016/j.dsx.2018.11.030 (2019).

18. Lee, S. B. et al. Triglyceride Glucose Index Is Superior to the Homeostasis Model Assessment of Insulin Resistance for Predicting Nonalcoholic Fatty Liver Disease in Korean Adults. Endocrinol Metab (Seoul), 34, 179-186 https://doi.org/10.3803/EnM.2019.34.2.179 (2019).

19. Kim, M. K. et al. Relationship between the triglyceride glucose index and coronary artery calcification in Korean adults. Cardiovasc Diabetol, 16, 108 https://doi.org/10.1186/s12933-017-0589-4 (2017).

20. Park, K. et al. Elevated TyG Index Predicts Progression of Coronary Artery Calcification., 42, 1569-1573 https://doi.org/10.2337/dc18-1920 (2019). 
21. Li, S. et al. The role of the triglyceride (triacylglycerol) glucose index in the development of cardiovascular events: a retrospective cohort analysis. Sci Rep, 9, 7320 https://doi.org/10.1038/s41598-019-43776-5 (2019).

22. Brito, A. D. M. et al. TAG-glucose (TyG) index in childhood: an estimate of cut-off points and the relation to cardiometabolic risk in 4- to 9year-old children. Public Health Nutr, 1-8 https://doi.org/10.1017/S1368980020000944 (2020).

23. Aldhoon-Hainerova, I. et al. Glucose homeostasis and insulin resistance: prevalence, gender differences and predictors in adolescents. Diabetol Metab Syndr, 6, 100 https://doi.org/10.1186/1758-5996-6-100 (2014).

24. Lee, J. H. et al. Incidence trends and associated factors of diabetes mellitus in Korean children and adolescents: a retrospective cohort study in Busan and Gyeongnam. Ann Pediatr Endocrinol Metab, 20, 206-212 https://doi.org/10.6065/apem.2015.20.4.206 (2015).

25. Kwon, E. B., Lee, H. S., Shim, Y. S., Jeong, H. R. \& Hwang, J. S. The changes of subtypes in pediatric diabetes and their clinical and laboratory characteristics over the last 20 years. Ann Pediatr Endocrinol Metab, 21, 81-85 https://doi.org/10.6065/apem.2016.21.2.81 (2016).

26. Castorani, V., Polidori, N., Giannini, C., Blasetti, A. \& Chiarelli, F. Insulin resistance and type 2 diabetes in children. Ann Pediatr Endocrinol Metab, 25, 217-226 https://doi.org/10.6065/apem.2040090.045 (2020).

27. Nogueira-de-Almeida, C. A. Metabolic syndrome definition in adolescents should incorporate insulin resistance. Ann Pediatr Endocrinol Metab, 25, 287-288 https://doi.org/10.6065/apem.2040166.083 (2020).

28. Buchanan, T. A., Watanabe, R. M. \& Xiang, A. H. Limitations in surrogate measures of insulin resistance. J Clin Endocrinol Metab, 95, 48744876 https://doi.org/10.1210/jc.2010-2167 (2010).

29. Borai, A., Livingstone, C. \& Ferns, G. A. The biochemical assessment of insulin resistance. Ann Clin Biochem, 44, 324-342 https://doi.org/10.1258/000456307780945778 (2007).

30. Simental-Mendia, L. E., Rodriguez-Moran, M. \& Guerrero-Romero, F. The product of fasting glucose and triglycerides as surrogate for identifying insulin resistance in apparently healthy subjects. Metab Syndr Relat Disord, 6, 299-304 https://doi.org/10.1089/met.2008.0034 (2008).

31. Vasques, A. C. et al. TyG index performs better than HOMA in a Brazilian population: a hyperglycemic clamp validated study. Diabetes Res Clin Pract, 93, e98-e100 https://doi.org/10.1016/j.diabres.2011.05.030 (2011).

32. Lee, S. H. et al. Predicting the development of diabetes using the product of triglycerides and glucose: the Chungju Metabolic Disease Cohort (CMC) study. PLoS One, 9, e90430 https://doi.org/10.1371/journal.pone.0090430 (2014).

33. Kim, J. W., Park, S. H., Kim, Y., Im, M. \& Han, H. S. The cutoff values of indirect indices for measuring insulin resistance for metabolic syndrome in Korean children and adolescents. Ann Pediatr Endocrinol Metab, 21, 143-148 https://doi.org/10.6065/apem.2016.21.3.143 (2016).

34. Detrano, R. et al. Coronary calcium as a predictor of coronary events in four racial or ethnic groups. N Engl J Med, 358, 1336-1345 https://doi.org/10.1056/NEJMoa072100 (2008).

35. Simental-Mendia, L. E. et al. The triglycerides and glucose index is associated with elevated blood pressure in apparently healthy children and adolescents. Eur J Pediatr, 178, 1069-1074 https://doi.org/10.1007/s00431-019-03392-x (2019).

36. Costa, R. F. et al. Metabolic syndrome in obese adolescents: a comparison of three different diagnostic criteria. J Pediatr (Rio J), 88, 303309 https://doi.org/10.2223/JPED.2200 (2012).

37. Yi, K. H. et al. Prevalence of insulin resistance and cardiometabolic risk in Korean children and adolescents: a population-based study. Diabetes Res Clin Pract, 103, 106-113 https://doi.org/10.1016/j.diabres.2013.10.021 (2014).

38. Kim, J. H. et al. The 2017 Korean National Growth Charts for children and adolescents: development, improvement, and prospects. Korean J Pediatr, 61, 135-149 https://doi.org/10.3345/kjp.2018.61.5.135 (2018).

39. Friedewald, W. T., Levy, R. I. \& Fredrickson, D. S. Estimation of the concentration of low-density lipoprotein cholesterol in plasma, without use of the preparative ultracentrifuge. Clin Chem, 18, 499-502 (1972).

40. A., R. R. \& M., S. D. Generalized additive models for location, scale and shape.Applied Statistics54,507-554, doi:https://doi.org/10.1111/j.1467-9876.2005.00510.x (2005).

\section{Figures}



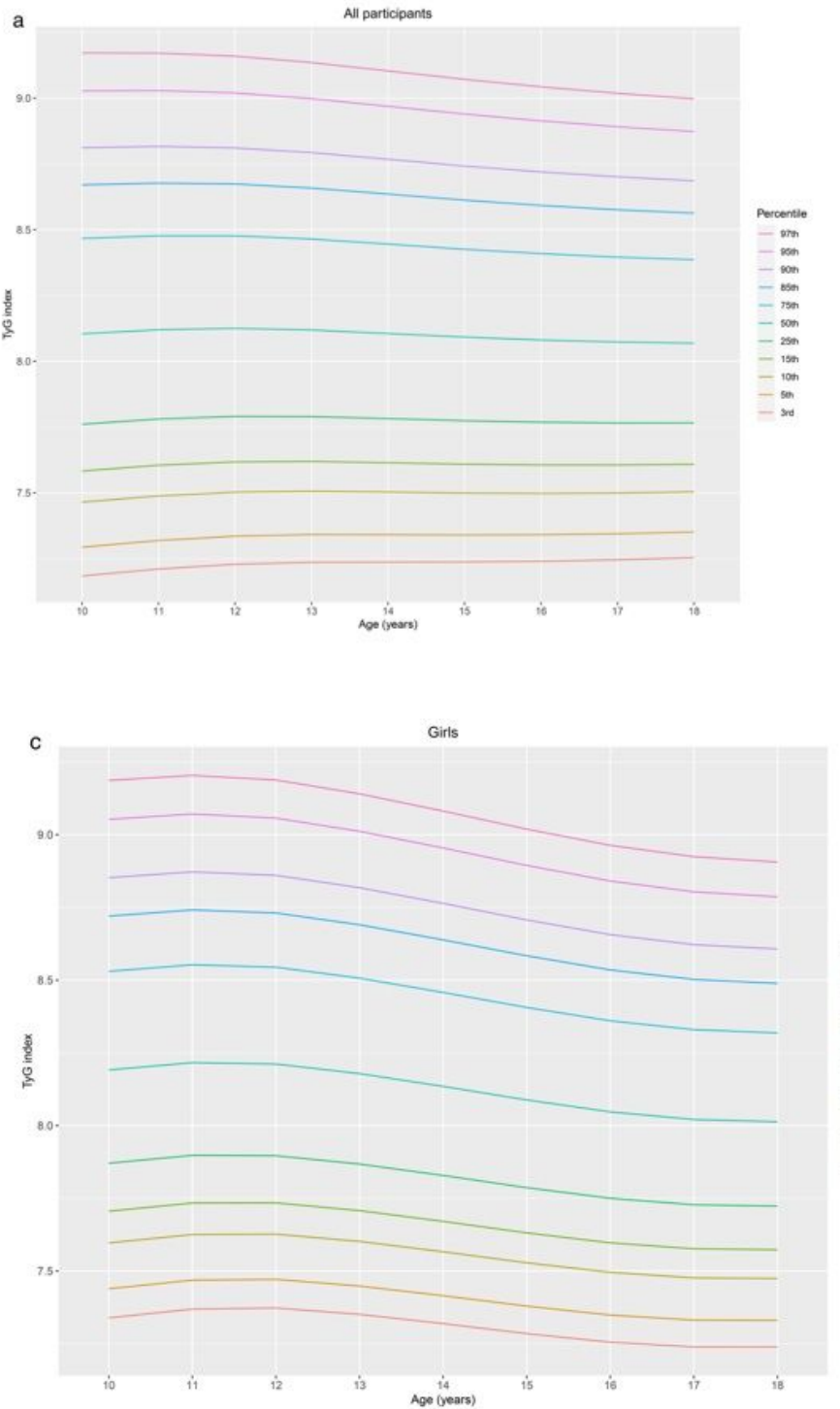

b

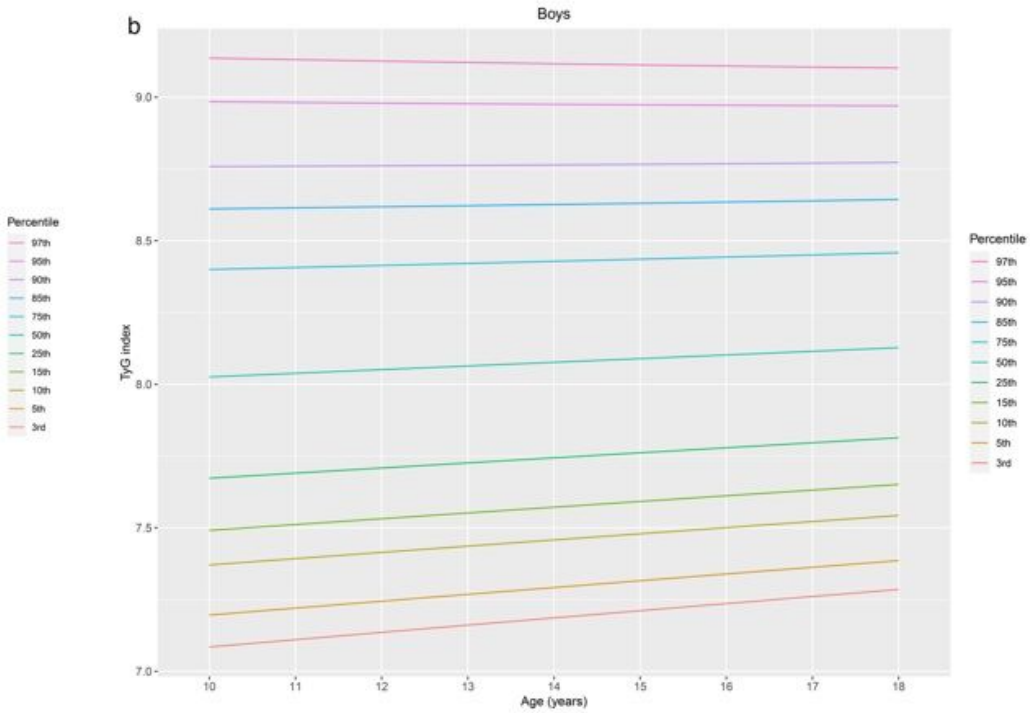

Figure 1

Percentile curves for the TyG index for all participants (a), boys (b) and girls (c) aged 10-18 years. The reference intervals for the age-specific TyG index are presented as the following percentile values: 3rd, 5th, 10th, 15th, 25th, 50th, 75th, 85th, 90th, 95th, and 97th, as indicated in different colors. TyG index, triglyceride-glucose index 